\title{
Dextrose prolotherapy for improvement of rotator cuff lesions: ready for clinical use?
}

Hong Kong Med J 2018;24:429-30

DOI: $10.12809 / \mathrm{hkmj} 187480$

To the Editor-Rotator cuff lesions (RCLs) are among the major causes of shoulder pain and dysfunction and affect the quality of life of many individuals including athletes, manual workers, and older adults. Most cases of RCLs, except for massive tears, are helped in the short term by conservative treatments such as rest, range of motion exercises, physiotherapy, analgesia, local corticosteroid injection, and minimally invasive therapeutic approaches. Chronic RCLs require more advanced invasive therapies to diminish shoulder pain and improve joint function. ${ }^{1}$

Prolotherapy is a minimally invasive regenerative therapy for chronic musculoskeletal disorders and tendinopathies. It involves injection of a nonbiological solution into the soft tissue or joint spaces. Hypertonic dextrose is the most commonly used prolotherapy solution. Tissue renewal and healing by injection of hypertonic dextrose, which is called dextrose prolotherapy (DP), has recently increased in popularity for treating musculoskeletal disorders and tendinopathies. It is believed that the injections can cause local tissue irritation by osmotic rupture of local cells and subsequently activate inflammatory responses. Thereafter, initiation of acute inflammatory responses can increase the proliferation of fibroblasts and collagen synthesis. This process can lead to healing and tissue renewal. ${ }^{2}$

Accordingly, recent studies have examined the effectiveness of DP on improvement of RCLs and reported some promising results. In a retrospective study with a 1-year follow-up period, Lee et $\mathrm{al}^{3}$ compared the efficacy of DP with conservative treatment in patients with RCLs. Their results revealed that DP improved shoulder pain, abduction, flexion, and external rotation with no adverse events. ${ }^{3}$ Although the study findings are promising, due to the nature of observational studies, it is difficult to conclude whether the observed association between DP treatment and improved outcome was related to that intervention. In a controlled trial, Bertrand et $\mathrm{al}^{4}$ evaluated the effects of dextrose and $0.1 \%$ lidocaine mixture along with physiotherapy on shoulder pain levels and degenerative changes of rotator cuff tendinopathy. The study solutions were injected at baseline, 1, and 2 months after the initiation of the study and the participants were followed up for 9 months. Results revealed that DP reduced shoulder pain and increase range of motion. However, DP failed to prevent or revert the degenerative changes of chronic rotator cuff tendinopathy. ${ }^{4}$ These findings are promising; however, the precise benefits of DP remain inconclusive because Bertrand et $\mathrm{al}^{4}$ used physical therapy along with DP in all studied groups without considering the control group. They also did not perform a covariance analysis to identify any significant effect of physical therapy on patient outcomes. In another clinical trial, Seven et $\mathrm{al}^{5}$ compared the effectiveness of DP versus physiotherapy in the treatment of pain and shoulder dysfunction in chronic RCLs. The authors found an improvement in the overall outcomes for both groups compared with baseline, but the intervention group had significantly lower pain and better shoulder range of motion in the first weeks of study. In addition shoulder abduction, flexion and internal rotation did not benefit from the DP intervention initially and shoulder external rotation did not benefit from DP at all. ${ }^{5}$

The discovery of new and innovative minimally invasive approaches should be a priority for orthopaedic surgeons. On the basis of the available evidence, DP may be useful for pain relief of RCLs along with current conservative treatment options. However, the limited available evidence on the efficacy of DP is inconclusive and further research is needed. Further well-designed clinical trials are warranted to determine the effectiveness of DP in patients with RCLs. In addition, the optimal concentration of dextrose, number of injections, time between injections, site and volume of injection, and follow-up period are yet to be determined.

\section{Declaration}

The author has disclosed no conflicts of interest. The author had full access to the data, contributed to the study, approved the final version for publication, and take responsibility for its accuracy and integrity.

\section{Reza Ganji *, MD}

Department of Orthopedic Surgery, School of Medicine, North Khorasan University of Medical Sciences, Bojnurd, Iran

*Corresponding author: r.ganji@nkums.ac.ir

\section{References}

1. Page MJ, Green S, McBain B, et al. Manual therapy and exercise for rotator cuff disease. Cochrane Database Syst Rev 2016;(6):CD012224.

2. Reeves KD, Sit RW, Rabago DP. Dextrose prolotherapy: 
a narrative review of basic science, clinical research, and best treatment recommendations. Phys Med Rehabil Clin N Am 2016;27:783-823.

3. Lee DH, Kwack KS, Rah UW, Yoon SH. Prolotherapy for refractory rotator cuff disease: retrospective casecontrol study of 1-year follow-up. Arch Phys Med Rehabil 2015;96:2027-32.
4. Bertrand $\mathrm{H}$, Reeves KD, Bennett CJ, Bicknell S, Cheng AL. Dextrose prolotherapy versus control injections in painful rotator cuff tendinopathy. Arch Phys Med Rehabil 2016;97:17-25.

5. Seven MM, Ersen O, Akpancar S, et al. Effectiveness of prolotherapy in the treatment of chronic rotator cuff lesions. Orthop Traumatol Surg Res 2017;103:427-33.

\title{
CORRECTIONS
}

\section{Correction to: YY Chee, Patrick HY Chung, Rosanna MS Wong, Kenneth KY Wong. Jaundice in infants and children: causes, diagnosis, and management.}

Hong Kong Med J 2018;24:285-92. DOI: 10.12809/hkmj187245.

Hong Kong Med J 2018;24:430

DOI: $10.12809 / \mathrm{hkmj} 187245-\mathrm{c}$

In Figure 5, on page 290 of the above article, a split path should be employed to the box 'Clinical picture consistent with breast milk jaundice and total serum bilirubin $<200 \mu \mathrm{mol} / \mathrm{L}$ '-a 'Yes' arrow to the box 'No further workup indicated/Anticipate spontaneous resolution by 12 weeks', and a 'No' arrow to the box 'Workup for haemolysis, possibility of Gilbert syndrome'.

The online article at www.hkmj.org has been corrected.

\section{Correction to: KM Yim, HF Ko, Marc LC Yang, TY Li, S Ip, J Tsui. A paradigm shift in the provision of improved critical care in the emergency department.}

\author{
Hong Kong Med J 2018;24:293-7. DOI: 10.12809/hkmj176902.
}

Hong Kong Med J 2018;24:430

DOI: $10.12809 / \mathrm{hkmj} 176902-\mathrm{c}$

The DOI, on page 293 of the above article, should have read '10.12809/hkmj176902' instead of '10.12809/hkmj166092'.

The online article at www.hkmj.org has been corrected. 